\title{
Deployment of Drone Base Station in Designated Area of a Campus Network
}

\author{
C. Ajaegbu ${ }^{1 *}$, O.O Adetunji', O. Awodele', S.O. Ogunlere' and R. U. Kanu² \\ 'Department of Computer Science ,Babcock University, llishan-Remo, Ogun State, Nigeria; ajaegbuc@babcock.edu.ng, \\ adetunjio@babcock.edu.ng, awodeleo@babcock.edu.ng, ogunleres@babcock.edu.ng \\ 2Department of Basic Science, Babcock University, Ilishan-Remo, Ogun State, Nigeria; \\ kanur@babcock.edu.ng
}

\begin{abstract}
Objectives: The study aimed at deploying Drone Base Station (DBS) within a selected area of a campus network and performed simulation using required parameters to ascertain performance level. Methods/Statistical Analysis: The researchers employed a circular placement method where the centre of the designed area was of utmost importance to ascertain, resulting to modelling of the drone base station deployment in a horizontal dimension. The total width of the building was also considered which formed a guide in determining the actual centre of the area under consideration, where altitude consideration was paramount. Findings: In the presented simulation figures, the horizontal distance between the drone base station and the user $(r)$ was tested using two different values. The two different values were considered and numerically established in consideration to the separate existing blocks within the campus high congestion area. It showed different result in the path loss in block A, while the coverage area for both values of $r$ appears to be same. However, it was observed that the altitude of coverage when $r$ is 20 is higher than when $r$ is 50 in the first simulation graph while block B and C showed slight difference which could be attributed to the change in their altitudes. This result also showed conformity with existing literature in the optimum deployment of DBS although deployment of DBS resulting to a kind of partitioning a closet has not really been tested within the cited literature. Thus, new frontier of managing effectively a macro cell and micro cell deployment within a closet like a campus network. Application/Improvement: The idea is encouraged in a campus/similar network with high traffic anddid not consider the possibility of interference. Hence, further studies in this direction.
\end{abstract}

Keywords: Base Station and Mobile Users, Campus Network, Deployment, Drone

\section{Introduction}

Theconstant increment in cellular network usage has called for attention towards maintaining a certain level of Quality of Service (QoS) to mobile users. This has called for various development stages in cellular network from the conventional base stations, to the small cell base station (femtocells) and now to the drone base station.

The advent of drone-cell is a future in cellular network as the terrestrial base station may not be able to render the satisfaction level required by cellular users. Although, DBS has its own limitations in terms of battery life which calls for limited time of operation ${ }^{1}$, its benefits as regards deployment flexibility and better communica- tion channels owing to the presence of short-range line of sight links ${ }^{1}$, is of utmost importance in the cellular world in support of the existing terrestrial base station, for improved QoS. Also, in DBS deployment, it is important to balance the association coverage with the traffic loads of the DBS in order to efficiently utilize its battery life while maintaining prompt delivery of data to mobile users $^{1,2}$. Though, many research works have been done on the optimal deployment of DBS and is still on going, it is also important to consider DBS deployment in a campus network as this could be seen as one of the centers of high traffic experienced by operators in any society. In this regard, the authors studied a campus network and its high traffic rate and proposed a DBS deployment in one

${ }^{*}$ Author for correspondence 
of the most strategic areas within the campus, known for high traffic congestion. The simulation output shows better coverage and reception with a high altitude of DBS. The rest of this paper is organized as follows: Section 2 presents literature review and related works. In Section 3, the system model, Section 4 shows the DBS deployment and its numerical parameters. Section 5 and 6 presents the result and conclusion respectively.

\subsection{Small Cell}

Small-cell systems are low powered cellular base stations designed to cover a certain portion of geographical location. This idea came majorly as the population rate of cellular users were outgrowing the macro base station, hence, leading to increase unavailability of radio resources when needed by cellular users. However, there is a need for a systematic network planning in order to manage effectively the increasing capacity of mobile subscribers. Small-cell systemsare primarily integrated in order to increase capacity in hot spots with high user demand and to augment for areas not covered by macro-cell base stations both outdoors and indoors. Their deployment has also aided the improvement of network performance and good quality of service by offloading from the large macro-cells. The combination of macro-cell and small cell results to a heterogeneous network providing increased bitrates per unit area ${ }^{3}$. See Figure 1. It is also important that when any operator is embarking on heterogenous form of deployment, the alloted portion for the service

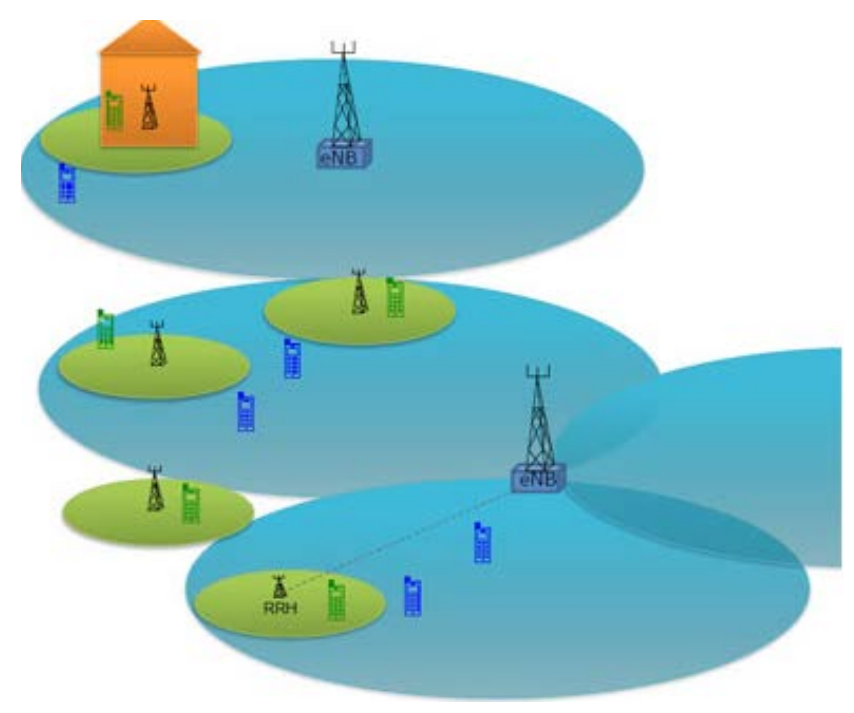

Figure 1. Illustration of a heterogeneous network of macro and small cells ${ }^{3}$. of small-cell should be substantial enough in order to maximize its deployment cost while utilizing its service effectively.

The antenna of a small cell coverage is placed above the madian but lower than the maximum height of the surrounding roof tops and its path loss determined with respect to the area/location of deployment. In mobile communication, classification of cell size is is solely dependent on $t$ maximum range of coverage where that of a small cell ranges between $1-3 \mathrm{~km}^{4}$.

Over the years, a good number of small-cell systems have emerged ranging from micro-cell, Pico cell to Femtocell. The various classifications is as a result of their respective sizes and range of coverage. Also, the following propagation losses are given below for small cell ${ }^{4}$.

For GSM 900 the corresponding propagation loss is given by: Loss $(\mathrm{dB})=132.8+38 \log (\mathrm{d} / \mathrm{km})$

For DCS 1800 the corresponding propagation loss is given by: $\operatorname{Loss}(\mathrm{dB})=142,9+38 \log (\mathrm{d} / \mathrm{km})$

For medium sized cities and suburban centres Loss $(\mathrm{dB})=145,3+38 \log (\mathrm{d} / \mathrm{km})$ for metropolitan centres.

\subsection{Microcells}

As stated by ${ }^{5}$, COST 231 defines microcell as a cell with it base station antenna mounted below roof top level where, its wave propagation is determined by diffraction and scattering around buildings. The following propagation model for a microcell with a free line of sight has been given as :

Path loss in dB $($ GSM 900 $)=101,7+26 \log (\mathrm{d} / \mathrm{km}) \mathrm{d}>20$ $\mathrm{m}$

Path loss in $\mathrm{dB}($ DCS 1800) $=107,7+26 \log (\mathrm{d} / \mathrm{km}) \mathrm{d}>$ $20 \mathrm{~m}$.

In microcell system, propagation loss is experienced in a high rate as the receiver moves away from line of sight of the transmitter. Microcell region ranges from 200 to 300 meters $^{7}$, hence, exhibit different usage patterns ranging from large and small cell system. Also, it could be supported by smaller and cheaper BTS ${ }^{8}$. This has also resulted to different BTS classes definitions, accommodating different microcell environments ${ }^{4}$.Technically, the frequency spacing in microcell specifications have been noted as 6 $\mathrm{MHz}$ between the microcell channels and other used cell channels within the vicinity. However, frequency spacing in microcell system is inversely proportional to Minimum 
Couple Loss (MCL) meaning that for smaller frequency spacings of $1.8 \mathrm{MHz}$, requires a larger MCL in order to guarantee successful close proximity operation ${ }^{8}$. This is so because of an increase in wideband noise and a decrease in the MS blocking requirement from mobiles closer to the carrier8.

\subsection{Picocell}

Pico in physics means a smaller unit of measurement when compared to micro. In essence, Pico cell refers to a system with smaller mobile cell coverage than micro. Meaning that the mobile coverage zone of a micro cell is larger than that of a Pico cell. Pico cell deployment is more to improving outdoor signals for mobile users while, saving battery life because of the short distance between the mobile users and the small cell system. A pico cell just like a femtocell simply takes a signal (either voice or data) from a macro base station and further extends it to a smaller range that is either lacking from coverage or experiencing a weak signal. Picocells are serviced and maintained and by a larger network provider while femtocells are usually designed to operate more autonomously when getting local feedback for radio frequency and protocol evaluation?.

In $^{11}$ asserted that different access policies could be used for a pico cell deployment such as: Open Access and Close access. In Open access, service provider placed the cell in a hotspot and all users can connect to it while in Close Access policy, the cell belongs to a user that wants all of the cell's capacity for him.

\subsection{Femtocell}

Femtocell is the most commonly used among the classes of small cell and as mentioned earlier, is designed to operate more autonomously when getting local feedback for radio frequency and protocol evaluation ${ }^{12}$. Femtocell popularity to industries began in the early in the year 2018 and since then has been used to offer solutions to some of the challenges faced by macrocell-based systems ${ }^{13}$. (Table 1) femtocell is self-installed by mobile users mostly for their own benefit in their home or office. The frequency and power level to operateon is solely determined by femtocells themselves rather than being dependent on the traditional method of site planning. This allows the network to adapt automatically as more femtocells are integrated or moved without the need for a complete frequency replan ${ }^{14}$.
Table1. Difference between femtocells and picocells ${ }^{12}$

\begin{tabular}{|l|l|}
\hline Femtocell & Picocell \\
\hline $\begin{array}{l}\text { Instalation is made by } \\
\text { customers }\end{array}$ & $\begin{array}{l}\text { Installation is made by } \\
\text { operators }\end{array}$ \\
\hline $\begin{array}{l}\text { Transmission to operator's } \\
\text { network is controlled by } \\
\text { customers }\end{array}$ & $\begin{array}{l}\text { Transmission to operator's } \\
\text { network is controlled by } \\
\text { operators }\end{array}$ \\
\hline $\begin{array}{l}\text { Frequency/radio parameters } \\
\text { is locally determined }\end{array}$ & $\begin{array}{l}\text { Frequency/radio parameters } \\
\text { is centrally planned }\end{array}$ \\
\hline $\begin{array}{l}\text { Site rental is the sole } \\
\text { responsibility of customers }\end{array}$ & $\begin{array}{l}\text { Site rental is sole } \\
\text { responsibility of operators }\end{array}$ \\
\hline
\end{tabular}

Femtocell industrial motivation came as a result the weak indoor signal experienced by mobile users while using $3 \mathrm{G}$ technology hence, femtocell architecture was designed with related $3 \mathrm{G}$ air interface protocol stack (Figure 2). Because of the promising future that femtocell has shown over these years, studies have gone further to improve both on the interference corrdination, security aspect and the general architecture towards being liable for expansion in order to accommodate more than one generation technology simultaneously. In $^{15}$ a cognitive radio that would be able to enhance interference coordination for femto cell networks was envisioned and presented to show better performance in handling cross-tier interference coordination with benchmarked with existing techniques.

In ${ }^{16}$ proposed demonstrated how femtocell architecture could be re-design to benefit developing countries where, their major focus was on African operators using both $2 \mathrm{G}$ and $3 \mathrm{G}$ technologies to transmit voice and data respectively (Figure 3 ). The major setback of femtocell is that, they would not normally broadcast a list of nearby neighbouring cells. Mobile phones could maintain a longlasting connection with femtocells but are faced with the challenge of call drops or short outage when a handover process is required from the mobile users ${ }^{12}$. It is true that femtocell and picocell play similar role in a smallcell sys-

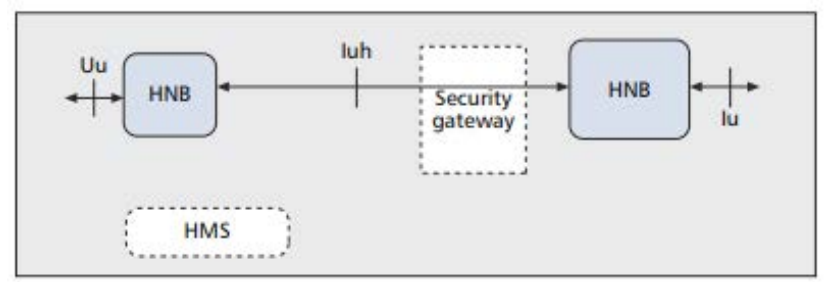

Figure 2. Femtocell architecture showing the luh air interface links ${ }^{13}$. 


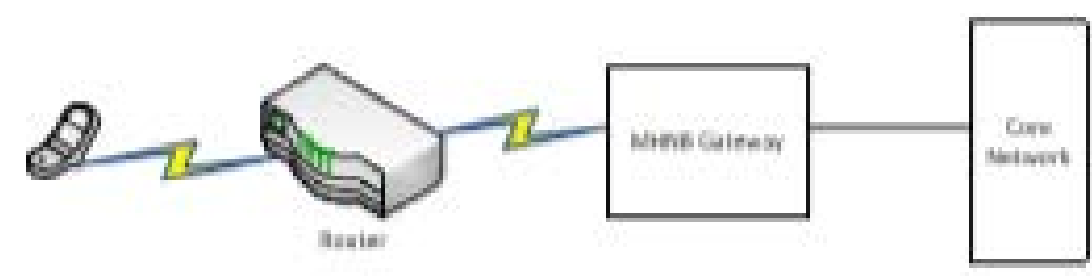

Figure 3. Block architecture of improved femtocell system ${ }^{16}$.

tem and have also been used interchangeably by some schorlarshowever; the table below shows some major difference between femtocell and picocell.

\subsection{Unmanned Aerial Vehicles}

Unmanned aerial vehicles commonly know as drone, has revolutionized the world of technology in the area of suivelliance, search and rescue mission, weather and traffic management and now the world of cullar network ${ }^{17}$.

As new innovation and ideas emerges, drone technology will continue to make impact in the world of technology. In cellular network, drone technology has taking over the small cell cellular technology and is continuing to offer more opportunities to operators and subscribers in terms of network management and quality of service respectively. Drone base station placement varieties have been considered by a good number of researchers: $\operatorname{In}^{1}$, the researchers looked at latency aware drone base station placement where one macro cell and one DBS were envisioned to coexist. The DBS placement problem was formulated as an optimization problem and LEAP algorithm was developed to provide solution ${ }^{2}$, envisioned an energy-efficient 3D placement of UAV with the goal of covering more users with minimum transmission power. They approached the placement problem by decoupling UAV-BS placement in the vertical dimension from the horizontal dimension. $\operatorname{In}^{18}$, the authors presented fairness aware multiple DBS deployment where two deployment scenarios were considered for the users and the DBS. Algorithms were proposed and evaluated for the two network different network scenarios. In ${ }^{19}$ presented an environment- aware DBS placement system were, more environmental information were utilized using ITU channel model. It was shown that by optimizing parameters selected by ITU model, the proposed model offered better performance in reduction of the root-mean-squared error compared to the widely used simpler model which does not total consider the ITU presented environmental factors. $\mathrm{In}^{20}$ envisioned a heuristic algorithm to deter- mine the number of DBS and their 3D locations needed to offer expected quality-of-service to a particular set of users with the assumption of no ground BS. $\mathrm{In}^{21}$ focused on improving spectral efficiency of drone small cells using algorithms to autonomously control the repositioning of drone in response to the users activity and movement. In ${ }^{22}$ investigated the effect of different wireless backhaul data rates on the number of served users were the wireless backhaul peak rate and bandwidth of the drone-BS were considered as the limiting factors for network centric and user centric approaches, in a heterogeneous network. Their study that the network centric approaches maximize the number of served users while user-centric maximized the sum-rate. In $^{23}$ proposed envisioned an energy efficient placement algorithm for drone base stations that should serve users using the minimum transmit power. Also, the optimal placement of the DBS was achieved in their work by decoupling the deployment problem in horizontal and vertical dimension. $\operatorname{In}^{24}$, the viability, fundamental performance and practical limitation of DBS as informative knowledge towards designing mobility control algorithms for drones that will ensure spectral efficiency of the floating base station was presented. $\operatorname{In}^{25}$ formulated a 3D-placement problem with the aim of maximizing revenue in the cellular network. In order to achieve their aim, they proposed a computationally efficient numerical solution. In ${ }^{26}$ noted the importance of energy efficient placement of DBS by proposing an optimal DBS placement method serving set of ground users using minimum required transmission power.

$\mathrm{In}^{27}$ proposed two clustering algorithms for dynamically placing 5G Unnamed Aerial Vehicles-Base Station by capturing the spatio-temporal relation between data demand points. In $^{28}$ studied the optimal deployment of two Drone Small Cells in both interference and interference free situations thereby, maximizing the coverage performance of Drone Small Cells (DSCs) through obtaining optimal values for DSC altitude and the distance between them. $\mathrm{In}^{29}$, proposed two algorithms 
that will determine the optimal height and solve placement challenges respectively with the aim of studying a novel 3D UAV-BS deployment that should maximize the number of covered users demanding different QoS requirements. $\operatorname{In}^{30}$, aimed at solving Drone-Base-Stations In-band full-duplex problem by proposing two heuristic algorithms based on different DBS placement by deploying two DBS system where one was a fixed DBS placement (used as benchmark) and the other, was a dynamic DBS placement aiming at improving performance. $\operatorname{In}^{31}$, investigated the issue of characterizing capacity limits of Unmanned aerial vehicles (UAV)-enabled multiuser communication system, by presenting a UAV-enabled two-user Broadcast Channel (BC), where a UAV moving at a constant altitude, is deployed to send independent information to two users at different fixed locations on the ground. $\mathrm{In}^{32}$, proposed a millimetre-Wave Aerial Base station to address the conventional issue of increasing traffic, experienced by the conventional terrestrial low power nodes deployment in the next generation of heterogeneous network. $\mathrm{In}^{33}$ studied the coexistence effect between ground and aerial nodes, thereby combining the propagation models for aerial links with the traditional stochastic propagation tool as used for cellular planning. $\mathrm{In}^{34}$ studied the coverage probability performance of multiple drone base stations and modelled drones distribution considering flexible dynamic changes using Poisson Point Process (PPP). In ${ }^{35}$, proposed a positioning algorithm for the DSCs deployment during emergency scenario where the old cellular network infrastructure was completely destroyed and users in need for a coverage rescue. $\mathrm{In}^{36}$ investigated the compatibility of UAVs with the conventional cellular infrastructure in the order of enabling high data rate requirements while improving the energy efficiency for the 5G and beyond systems. In $^{37}$ developed a novel framework simulator that exploits geographical information from google earth engine, enabling environment aware deployment of multiple drone base stations towards providing optimal connectivity to cellular ground users. $\operatorname{In}^{38}$, multiple drone-mounted base stations were designed to operate freely with the help of a mobility control algorithm in order to serve mobile users on the ground, thereby reducing the distance between the base stations and the users. In ${ }^{39}$, a spectral efficiency was derived for delivering traffic (messages) from the macro base station to a ground user via drone-mounted base station, which is operated in the half-duplex in-band mode. The spectra efficiency was maximized using the Spectral efficient Aware DBS placement and user (STABLE) algorithm.

Provided a method for a seamless replacement of a drone base station with a second drone base station by sending pilot signals to and fro the two drones. $\operatorname{In}^{40}$, a new mobility model was developed for drone base stations where they can move freely ignoring the cell boundariesthis was validated by evaluating the performances of two user association scheme. $\mathrm{In}^{41}$, the researchers investigated an underlay spectrum sharing between the 3D Drone Small Cells (DSC) and the traditional cellular networks modeled by the $2 \mathrm{D}$ Poisson point processes, the analytical and numeric results of the model showed that the maximum throughput of the DSCs user increases almost linearly with the increase of the DSCs outage constraint. $\mathrm{In}^{42}$ addresses the possibility of deploying drone base stations and focuses on the practical limitations of drones to be used in cellular networks. An autonomous mobility control algorithm was developed to improve the spectral efficiency of the floating base stations. $\operatorname{In}^{43}$ the researchers modeled the effect of interference coming from coexisting ground networks on the aerial link which could be the uplink of an aerial cell served by a drone base station. The benefits obtained by a drone from a better line of sight were demonstrated.

\section{System models/Methodology}

Consider a congested macrocell serving an entail community including a campus within a rural setting (but with urban planning) where the biggest building on the campus is always experiencing congestion due to heavy traffic occurring within the hours of 9am - 5pm working days and during some academic events. Let $\mathrm{M}$ represents the set of mobile users competing for mobile channels from the Macro Base Station (MBS) with the total MU's data rate per hour denoted as $r_{i}^{\mathrm{m}}$ modified from ${ }^{1}$ as:

$$
r_{i}^{m}=w_{m} \log _{2}\left(1+\frac{p_{m} g_{m}}{N}\right)
$$

Where wm is the total amount of bandwidth available from the MBS and Pm Maximum transmission power of the MBS, gm represents the channel gain between the $\mathrm{MBS}$ and the $\mathrm{MU}$ while $\mathrm{N}$ represents the various dynamic campus signal environmental impediments. Also, let the subsets of $\mathrm{M}$ be denoted as $\mathrm{i}$, this implies that each mobile user occupies a location of (xi,yi) and hi represents the 
possible placement altitudes of the DBS hence the radio signals emitted by DBS (air-to- ground channels) as noted $i^{2}$, are mainly Line-of-Sight (LoS) or non-Line-of-Sight (NLoS) where the probability of having LoS connection between the mobile users and the DBS has been adopted from $^{2}$ as:

$$
P_{\text {LoS }}=\frac{1}{1+a \exp \left(-b\left(\frac{180}{\pi} \tan ^{-1}\left(\frac{h}{r_{i}}\right)-a\right)\right)}
$$

Where $a$ and $b$ as noted in [17] are constants dependent on environmental factors and

$$
r_{i}=\sqrt{\left(x_{i}-x_{D}\right)^{2}+\left(y_{i}-y_{D}\right)^{2}}
$$

Where, $\left(\mathrm{x}_{\mathrm{D}}, \mathrm{y}_{\mathrm{D}}\right)$ represents the location of the DBS. Again, the probability of the Non-line of sight (NLoS) is given by

$$
P_{\text {NLoS }}=1-P_{L o S}
$$

Since in this work the major focus is to determine the efficient placement point that will yield optimum radius coverage, we adopted the distance between the user and the DBS (denoted as di) from ${ }^{2}$ as a measuring metric against the coverage region of the entire building. Hence, it is given as:

$$
d i=\sqrt{h i^{2}+r i^{2}}
$$

\section{Deployment of Drone Base Station}

The building presents the centre of majority of activities each day and also containing $70 \%$ of the total department in the campus. Hence, every operational hour experiences heavy congestion on a normal daily activity and extremely congested during occasional events like graduation or matriculation ceremony. In such case, the terrestrial base station experiences difficulty serving all its users. In order to maintain a good quality of service to the users within and inside the vicinity of the building, we consider the deployment of a drone base station serving as a microcell within the vicinity with a backhaul connection to the macrocell. The idea is to study the best DBS placement altitude level that will offer an optimum coverage with minimum path loss to a campus main event building. The various altitude levels that were subjected into examination cut across: slightly above roof-top, rooftop level and also established literature altitudes. The study also adopted the probabilistic model based on the height of the building. The campus main event building is made up of three Blocks: Block A, B and C as depicted in Figure 1, 4 and 2 respectively with Block A recording the highest height among other blocks.

$\mathrm{In}^{6,19}$, it was noted that the probabilistic model depends on the combination of free-space loss with excessive loss as a result of environmental impediments. With the probability of LOS given above, the free- space loss as recorded by Friis Equation in ${ }^{6,19}$ is given as:

$$
L_{f}(h, r)=20 \log \left(4 \pi f_{c} / c\right)+20 \log (d) d B
$$

And the excessive loss given as:

$$
L_{e}(h, r)=P_{\pi L O S}+\left(1-P_{\pi N L O S}\right) d B
$$

Where, л is used to denote excessive loss due to diffraction and reflection ${ }^{6,19}$. Hence the total loss becomes ${ }^{6,19}$ :

$$
L_{f}(h, r)+L_{e}(h, r)
$$

Since the aim is to measure the coverage area with minimum pathloss, the coverage radius is determined at each point of altitude variation using the Equation:

$$
R=A / h
$$

Where, $\mathrm{R}$ is the DBS coverage radius and $\mathrm{A}$ is the total area of the surrounding. Thus, Table 2 shows the parameters for channel model.

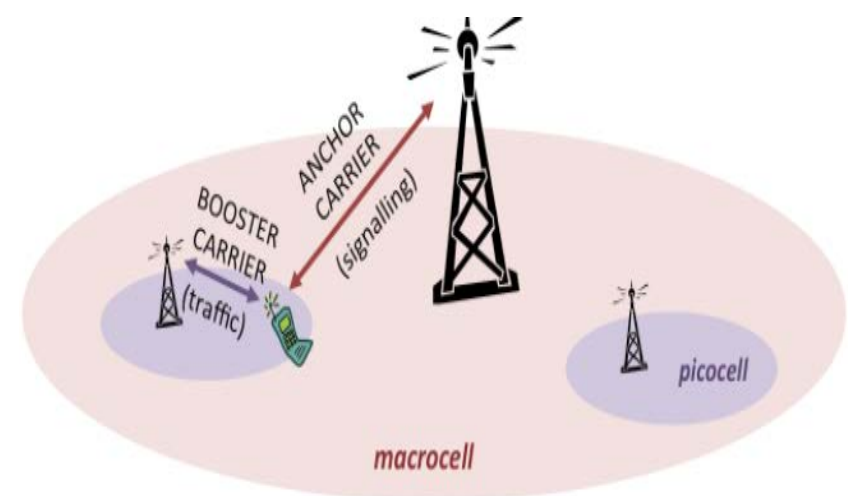

Figure 4. Operating principle of a Picocell ${ }^{10}$. 
Table 2. Parameters for channel model

\begin{tabular}{|c|c|}
\hline Parameters & Values \\
\hline Carrier frequency $\left(f_{c}\right)$ & $\begin{array}{l}2.6 \mathrm{GHz} \text { (from Nigeria } \\
\text { Communication commission) }\end{array}$ \\
\hline $\begin{array}{l}\text { Width of blocks with } \\
\text { (Including the back space) }\end{array}$ & $63,437 \mathrm{~mm}$ \\
\hline Height of Block A with roof & $14,810 \mathrm{~mm}$ \\
\hline $\begin{array}{l}\text { Height of Block B and C } \\
\text { with roof }\end{array}$ & $13,250 \mathrm{~mm}$ \\
\hline $\mathrm{a}$ and $\mathrm{b}$ & 9.61 and 0.16 respectively [5] \\
\hline лLOS (high-rise) & $2.3 \mathrm{~dB}[4]$ \\
\hline лNLOS (high-rise) & $34 \mathrm{~dB}[4]$ \\
\hline $\begin{array}{l}\text { Altitudes }(\mathrm{h}) \text { under } \\
\text { consideration for Block B } \\
\text { and C }\end{array}$ & $\begin{array}{l}50 \mathrm{~m}, 100 \mathrm{~m}, 600 \mathrm{~m}, 1000 \mathrm{~m} \\
{[5,4 \& 6] \text { and } 14000 \mathrm{~mm},} \\
13250 \mathrm{~mm} \text { [field measurement } \\
\text { heights] }\end{array}$ \\
\hline $\begin{array}{l}\text { Altitudes (h) under } \\
\text { consideration for Block A }\end{array}$ & $\begin{array}{l}50 \mathrm{~m}, 100 \mathrm{~m}, 600 \mathrm{~m}, 1000 \mathrm{~m} \\
{[5,4 \& 6] \text { and } 15000 \mathrm{~mm},} \\
14810 \mathrm{~mm} \text { [field measurement } \\
\text { height] }\end{array}$ \\
\hline
\end{tabular}

\section{Simulations and Discussions}

The parameters presented in Table 2 were used for the modelling of the campus drone placement to ascertain its performance in terms of coverage area and path loss. Figure 3, shows the simulation result of the coverage area and path loss of Block A of the section of the campus under congestion. In the figure, the horizontal distance between the drone base station and the user $(r)$ was tested using two different values. The two different values were considered and numerically established in consideration to the separate existing blocks within the campus high congestion area. It shows that the path loss in block A when $r$ is 20 , is higher compared to when $r$ is 50 while the coverage area for both values of $r$, appears to be same. Again, it was observed that the altitude of coverage when $r$ is 20 is higher than when $r$ is 50 .

Figure 5 shows the simulation result of the coverage area and path loss as occurs in block B and C (Figure 6), (Figure 7) and (Figure 8). It was observed that similar result of the block A (Figure 9) occurred only with a slight difference which could be attributed with the change in their altitudes (as obtained in the field experiment). Furthermore, the coverage area in Figure 4, increased respectively for each of the different values of $r$ as compared with Figure 3. Also, the altitude of Figure 5 when $r$ is 20 appears to extend much higher as this will offer

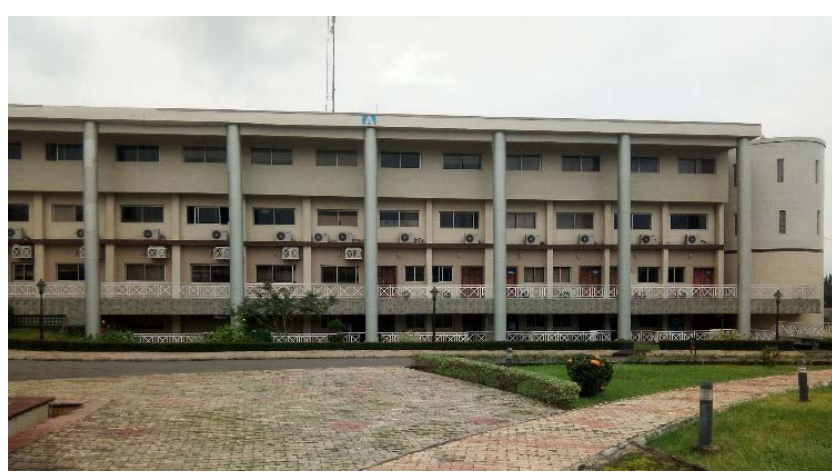

Figure 5. Block A (reserchers's specimen, 2019).

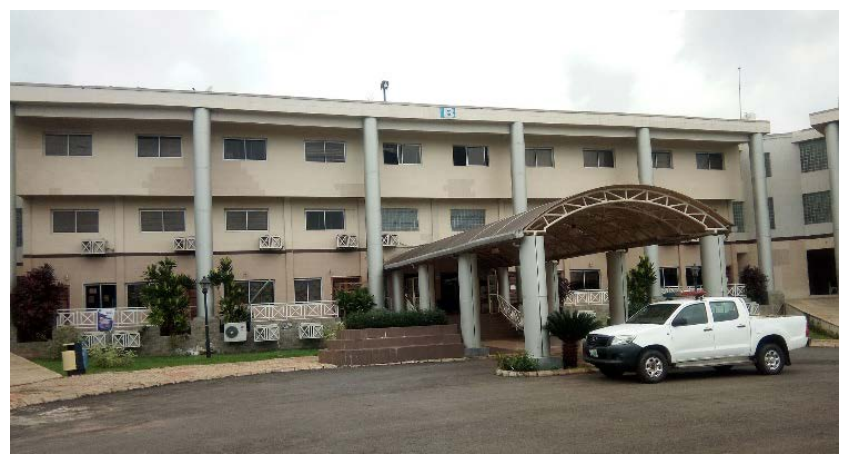

Figure 6. Block B (reserchers' specimen, 2019).

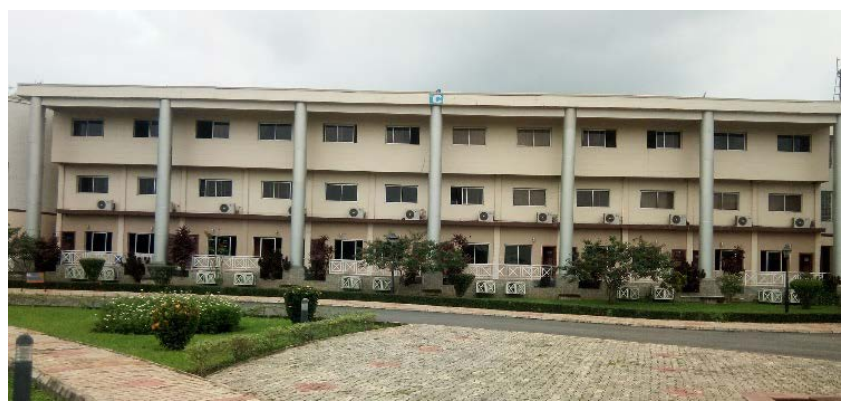

Figure 7. Block C (reserchers' specimen, 2019).

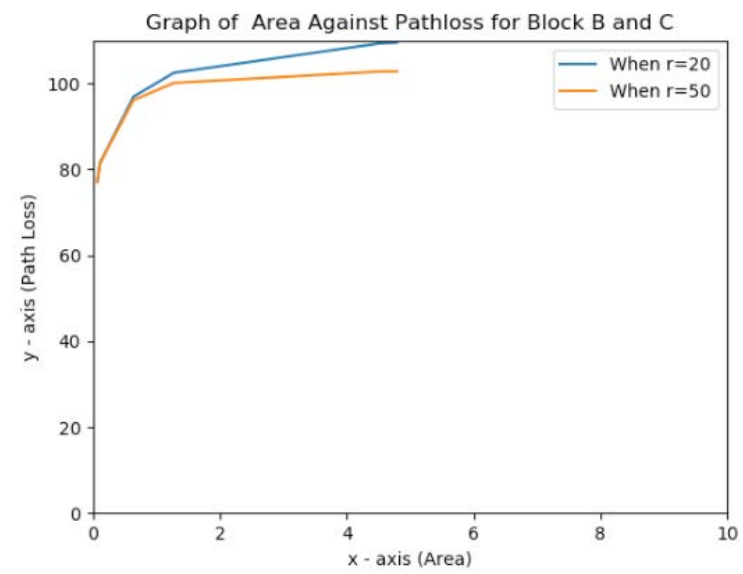

Figure 8. Plot of path loss against coverage area of Block B \& C. (reserchers' result, 2019). 


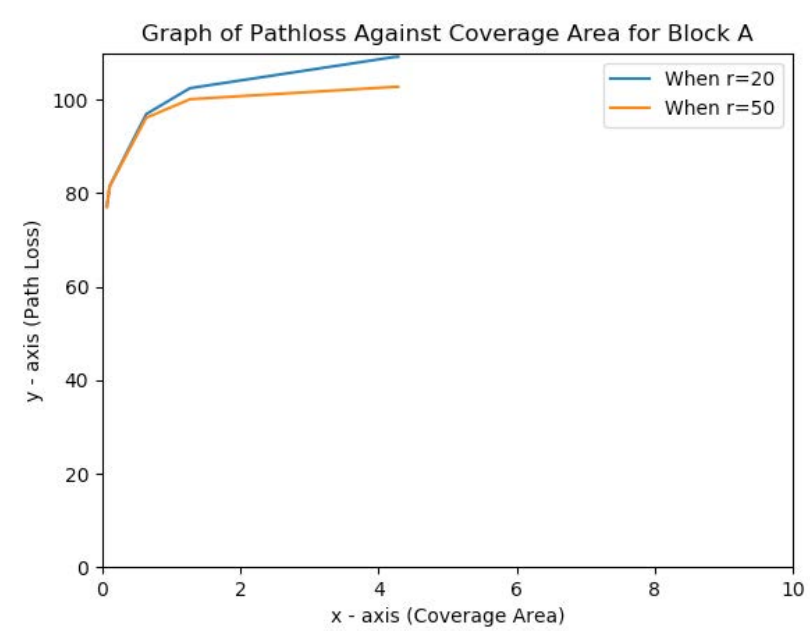

Figure 9. Plot of path loss against coverage area of BlockA (reserchers' result, 2019).

better reception to users in higher floors compared to the result obtained in Figure 3.

\section{Conclusions}

This studyshows drone base station deployment and its performance of a selected location within a campus environment with higher rate of congestion both during the working hours and occasional events.

The simulation output shows better coverage and reception with a high altitude of DBS and hence, proposes such deployment integration planning between macro cell and DBS for similar campus structuring and layout. In the future work, the researchers intend developing an energy efficient routing protocol for DBS deployments.

\section{References}

1. Latency aware drone base station placement in heterogeneous networks. Available from: https://arxiv.org/ abs/1710.01126

2. Alzenad M, El-Keyi A, Lagum F, Yanikomeroglu H. 3D placement of an unmanned aerial vehicle base station (UAV-BS) for energy-efficient maximal coverage. IEEE Wireless Communication Letters. 2017; 6(4):434-7. https:// doi.org/10.1109/LWC.2017.2700840

3. The mobile Broadband Standard. Available from: http:// www.3gpp.org/hetnet

4. Digital cellular telecommunications system (Phase 2+); Radio network planning aspect (3GPP TR 43.030 version 12.0.0 Release 12). Available from: https://www.etsi. org/deliver/etsi_tr/143000_143099/143030/12.00.00_60/ tr_143030v120000p.pdf

5. Digital cellular telecommunications system (Phase 2+); Radio link management in hierarchical net-works (3GPP TS 45.022 version 8.0.0 Release 8). Available from: https:// www.etsi.org/deliver/etsi_ts/145000_145099/145022/08.00 .00_60/ts_145022v080000p.pdf

6. Digital cellular telecommunications system (Phase 2+); Radio network planning aspects (3GPP TR 43.030 version 6.1.0 Release 6). Available from: https://www.etsi. org/deliver/etsi_tr/143000_143099/143030/06.01.00_60/ tr_143030v060100p.pdf

7. Digital cellular telecommunications system (Phase 2+); Radio network planning aspects (GSM 03.30 version 7.0.0 Release 1998). Available from: https://www.etsi. org/deliver/etsi_tr/101300_101399/101362/07.00.00_60/ tr_101362v070000p.pdf

8. Digital cellular telecommunications system (Phase 2+); Radio network planning aspects (3GPP TR 43.030 version 8.0.0 Release 8). Available from: https://www.etsi. org/deliver/etsi_tr/143000_143099/143030/08.00.00_60/ tr_143030v080000p.pdf

9. Picocell. Available from: https://www.techopedia.com/definition/16860/picocell

10. Enhanced small cells are the key to traffic boom. Available from: https://www.unwiredinsight.com/tag/picocells

11. Picocell positioning in an LTE network. Available from: https://fenix.tecnico.ulisboa.pt/downloadFile/395145917062/resumo.pdf

12. What's the difference between picocells and femto cells. Available from: https://www.thinksmallcell.com/FAQs/ whats-the-difference-between-picocells-and-femtocells. html

13. Douglas KN, Yoshizawa T, Favichia F. Standardization of Femtocells in 3GPP. IEEE Communica-tions Magazine.2009; 47:68-75. https://doi.org/10.1109/MCOM.2009.5277458

14. Femtocells- Architecture and Network Aspects. file://C:/ Users/a/Downloads/qualcomm-researc h-small-cellsarchitecture-network-aspects.pdf. Date accessed: 28/01/2010.

15. Wang W, Yu G, Huang A. Cognitive radio enhanced interference coordination for femtocell net-works. IEEE Communications Magazine. 2013; 51(6):37-43. https://doi. org/10.1109/MCOM.2013.6525593

16. Ajaegbu C, Awodele O, Aloa OD. A multi-mode home node-b air interface protocol stack model: an opportunity for African Countries. An International Journal of Advanced Computer Technology. 2018; 7(9):2820-5.

17. Understanding UAV cellular communications: from existing networks to massive MIMO. Available from: https:// arxiv.org/abs/1804.08489 
18. Akarsu A,Girici T. Fairness aware multiple drone base station deployment. IET Communications. 2018; 12(4):425-31. https://doi.org/10.1049/iet-com.2017.0978

19. Bor-Yaliniz I, Szyszkowicz SS, Yanikomeroglu H. Environment-aware drone-base-station place-ments in $\mathrm{m}$ odern metropolitans. IEEE Wireless Communications Letters. 2018; 7(3):372-5. https://doi.org/10.1109/ LWC.2017.2778242

20. Kalantari E, Yanikomeroglu H, Yongacoglu A. On the number and 3D placement of drone base sta-tions in wireless cellular networks. IEEE 84th Vehicular Technology Conference (VTC-Fall); 2016. p. 1-6.

21. Fotouhi A, Ding M, Hassan M. Dynamic base station repositioning to improve performance of drone small cells. 2016 IEEE Globecom Workshops (GC Wkshps); 2016. p. 1-6.

22. Kalantari E, Shakir MZ, Yanikomeroglu H,Yongacoglu A. Backhaul-aware robust 3D drone Place-ment in 5G+ wireless networks. IEEE International Conference on Communications Workshops (ICC Workshops); 2017. p. 109-14.

23. Wang L, Hu B, Chen S. Energy efficient placement of a drone base station for minimum required transmit power. IEEE Wireless Comunication Letters. 2018; 1-1.

24. Fotouhi A. Towards intelligent flying base stations in future wireless network. IEEE 18th Interna-tional Symposium on a World of Wireless, Mobile and Multimedia Networks (WoWMoM); 2017. p. 1-3. https://doi.org/10.1109/ WoWMoM.2017.7974302

25. Efficient 3-D placement of an Aerial Base Station in next generation cellular networks. Available from: https://arxiv. org/abs/1603.00300

26. Wang L, Hu B, Chen S. Energy efficient placement of a drone base station for minimum required transmit power. IEEE Wireless Communication Letters. 2018. https://doi. org/10.1109/LWC.2018.2808957

27. Iellamo S, Lehtomaki JJ, Khan Z. Placement of $5 \mathrm{G}$ drone base stations by data field clustering. IEEE 85th Vehicular Technology Conference (VTC Spring); 2017. p. 1-5.

28. Mozaffari M, Saad W, Bennis M, Debbah M. Drone Small cells in the clouds: design, deployment and performance analysis. IEEE Global Communications Conference (GLOBECOM); 2015. p. 1-6. https://doi.org/10.1109/ GLOCOM.2015.7417609

29. 3D placement of an unmanned aerial vehicle base station for maximum coverage of users with dif-ferent QoS requirements. Available from: https://arxiv.org/abs/1709.05235

30. Zhang L, Fan Q, Ansari N. 3-D drone-base-station placement with in-band full-duplex communica-tions. IEEE
Communications Letters. 2018; 22(9):1902-5. https://doi. org/10.1109/LCOMM.2018.2851206

31. Capacity characterization of UAV-enabled two-user broadcast channel. Available from: https://arxiv.org/ abs/1801.00443

32. Zhang T, Zhou F, Feng L, Yu P, Li W, Rong B, Rutagemwa $H$. Capacity enhancement for next gen-eration mobile networks using mmwave aerial base station. IEEE Global Communications Confe-rence; 2017. p. 1-6.

33. Coexistence of terrestrial and aerial users in cellular networks. Available from: https://arxiv.org/abs/1710.03103

34. Coverage maximization for a poisson field of drone cells. Available from: https://arxiv.org/abs/1708.06598

35. Klaine PV, Nadas JPB, Souza RD, Imran MA. Distributed drone base station positioning for emer-gency cellular networks using reinforcement learning. Cognitive Computation.2018;10(5):790-804.https://doi.org/10.1007/ s12559-018-9559-8 PMid:30363787 PMCid:PMC6182572

36. Raza Naqvi SA, Hassan SA, Pervaiz H, Ni Q. Droneaided communication as a key enabler for $5 \mathrm{G}$ and resilient public safety networks. IEEE Communication Magazine. 2018; 56(1):36-42. https://doi.org/10.1109/ MCOM.2017.1700451

37. Environment-aware deployment of wireless drones base stations with Google Earth simulator. Available from: https://arxiv.org/abs/1805.10424

38. Fotouhi A, Ding M, Hassan M. Flying drone base stations for macro hotspots. IEEE Access. 2018,6, pp. 19530-19539. https://doi.org/10.1109/ACCESS.2018.2817799

39. Sun X, Ansari N. Jointly optimizing drone-mounted base station placement and user association in heterogeneous networks. IEEE International Conference on Communications (ICC); 2018. p. 1-6. https://doi. org/10.1109/ICC.2018.8422377

40. Service on Demand: Drone Base Stations Cruising in the Cellular Network. Available from: https://arxiv.org/ abs/1710.09504

41. Zhang C, Zhang W. Spectrum sharing for drone networks. IEEE Journal on Selected Areas in Communications. 2017; 35(1):136-44.

42. Fotouhi A. Towards intelligent flying base stations in future wireless network. IEEE 18th Interna-tional Symposium on A World of Wireless, Mobile and Multimedia; 2017. p. 1-3. https://doi.org/10.1109/WoWMoM.2017.7974302

43. Uplink performance analysis of a drone cell in a random field of ground interferers. Available from: https://arxiv. org/abs/1711.01351 